\title{
Discrete dislocation plasticity analysis of the effect of interfacial diffusion on the creep response of Ni single-crystal superalloys
}

\author{
Siamak S. Shishvan ${ }^{\mathrm{a}, \mathrm{b}}$, Robert M. McMeeking, ${ }^{\mathrm{c}, \mathrm{d}}$, Tresa M. Pollock ${ }^{\mathrm{e}}$, Vikram S. Deshpande,** \\ ${ }^{a}$ Department of Structural Engineering, University of Tabriz, P.O. Box 51666-16471, Tabriz, Iran \\ ${ }^{b}$ Department of Engineering, University of Cambridge, Trumpington Street, Cambridge CB2 1PZ, UK \\ ${ }^{c}$ Department of Materials and Department of Mechanical Engineering, University of California Santa Barbara, CA \\ 93106, USA \\ ${ }^{d}$ School of Engineering, University of Aberdeen, King's College, Aberdeen AB24 3UE, UK \\ ${ }^{e}$ Department of Materials, University of California Santa Barbara, CA 93106-5050, USA
}

\begin{abstract}
Discrete dislocation plasticity (DDP) analysis of the high temperature creep deformation of a single crystal Ni superalloy comprising $\mathrm{Ni}_{3} \mathrm{Al}$ precipitates $\left(\gamma^{\prime}\right)$ in a Ni matrix $(\gamma)$ is presented. The $\gamma^{\prime}$ precipitates remain elastic but can also deform due to the stress-driven inter-diffusion of the Al within the Ni on the $\gamma / \gamma^{\prime}$ interface while plastic deformation of the $\gamma$ phase occurs by a combination of dislocation glide and dislocation climb coupled to the diffusion of vacancies. At relatively low applied uniaxial tensile stresses, the creep strain rates are very low in the absence of interfacial diffusion. This is due to the stress-induced pile up of dislocations at $\gamma / \gamma^{\prime}$ interfaces that serves to inhibit further nucleation and suppresses continued plastic flow in the $\gamma$ phase. When interfacial diffusion is permitted, the creep rates not only increase but the superalloy also exhibits distinct secondary and tertiary creep regimes. While this change in behaviour is a result of interfacial diffusion, the contribution of the average $\gamma^{\prime}$ strain to the deformation of the superalloy is small. Rather, the diffusional deformation at the interface results in the development of a wavy interface which relaxes the back-stresses of dislocations piled-up at the $\gamma / \gamma^{\prime}$ interfaces. This permits continued dislocation activity within the $\gamma$ phase with dislocations arranging themselves into low energy cell-structures in the $\gamma$ phase via dislocation climb. The formation of these structures results in an increase in the creep strain rate and the onset of the tertiary creep regime. At high applied stresses, the high initial dislocation density within the $\gamma$ phase results in the continued climb motion of dislocations and an evolving spatial distribution of vacancies within the superalloy. Thus, creep deformation occurs even in the absence of interfacial diffusion although the creep rates are significantly increased when interfacial diffusion is present. The DDP analysis presented here demonstrates the critical role of interfacial diffusion in controlling the creep rates of Ni superalloys and suggests that interface engineering to reduce interfacial diffusion rates will aid in improving the creep performance of these alloys.
\end{abstract}

Keywords: single-crystal superalloys, creep deformation, interfacial diffusion, discrete dislocation plasticity

\footnotetext{
*Corresponding author

Email addresses: sshishvan@tabrizu. ac. ir (Siamak S. Shishvan), rmcm@engineering. ucsb . edu (Robert M. McMeeking), pollock@engineering.ucsb.edu (Tresa M. Pollock), vsd@eng. cam.ac.uk (Vikram S. Deshpande )
} 


\section{Introduction}

The creep response of nickel-based single crystal superalloys often controls the service life of blades used in aircraft and gas turbine engines. Their microstructure can be very crudely approximated as a two-phase brick-and-mortar composite comprising a high volume fraction of the strengthening $\mathrm{Ni}_{3} \mathrm{Al}$ precipitate (denoted $\gamma^{\prime}$ ) in a single crystal Ni matrix (denoted $\gamma$ ). Moreover, a number of alloying elements are added to enhance relevant properties. For example, $\mathrm{Cr}$ and $\mathrm{Co}$ are added to improve environmental resistance while Mo, W, Re and possibly Ru added for creep strengthening; see Pollock and Tin [1] for further details. Thus, these alloys have complex microstructures and chemical compositions that largely are chosen by extensive experimental testing and metallurgical rules-of-thumb derived from experience. More recently, the desire to improve the design methodologies of these alloys has led to significant advances connecting micro-scale mechanisms to macro-scale deformation of precipitate- strengthened alloys [2] and thereby models for the composition dependence of the creep deformation behaviour of nickel-based superalloys

[3]. However, most of these models employ a number of empirical relations (e.g. the Orowan equation) with the applicability of such macroscopic relations in the nano-meter size $\gamma$ channels remaining unclear.

The creep deformation behavior of superalloy single crystals varies substantially over the relevant range of stress and temperature for these materials [1, 4]. At low temperature and high stress $\left(T<\sim 800{ }^{\circ} \mathrm{C}\right.$ and applied stress $>\sim 700 \mathrm{MPa}$ ) dislocation shearing of the ordered precipitates is prominent and primary creep strains are a dominant feature of the creep curve. At high temperature and low stress $\left(T>\sim 1000{ }^{\circ} \mathrm{C}\right.$ and applied stress $\left.<\sim 200 \mathrm{MPa}\right)$ stress-induced directional coarsening occurs, converting individual precipitates into high aspect ratio plates ("rafts") that obstruct the dislocation glide in the matrix. Under these conditions, a brief, low strain primary creep transient is followed by an extended period of steady state creep. Over the wide remaining intermediate temperature and stress range, primary creep strains are limited and creep rates continuously accelerate, with an extended tertiary transient that is not influenced by creep damage (voids, cracks, recrystallization) until the very late stages of life. In this particularly important intermediate regime the stresses are lower than that necessary to cut the $\gamma^{\prime}$ precipitates but the temperature 
is high enough so that primary creep is precluded, thus dislocations glide through the $\gamma$ phase and are then obstructed by the $\gamma / \gamma^{\prime}$ interfaces. Plastic flow rates are thus controlled by the climb rate of dislocations, with a limited influence of solutes on the glide processes. Therefore, the creep response of the brick-and-mortar superalloy microstructure is controlled by the climb-assisted glide of dislocations which to date remains poorly understood. In addition, the development of a wavy $\gamma / \gamma^{\prime}$ interface during creep deformation in the tertiary regime has also been reported; see for example $[5,6]$. This has been attributed to the inter-diffusion of the $\mathrm{Al}$ in the vicinity of the $\gamma / \gamma^{\prime}$ interface, driven by elastic strain energy of the dislocations near the $\gamma / \gamma^{\prime}$ interface. The effect of this interfacial diffusion mechanism on the creep response of the superalloy remains unclear.

There have been significant advances in discrete dislocation plasticity (DDP) modelling approaches wherein plastic flow is via climb-assisted glide motion of dislocations. For example Raabe [7] outlined a formulation for including climb in discrete dislocation calculations and then employed this idea to model creep of nickel-based superalloys in [8]. Subsequently, Davoudi et al. [9] and Danas and Deshpande [10] extended these ideas to enable the solution of boundary value problems. However, all these studies have used some version of a drag-type relation for the climb motion of dislocations based on the quasi steady-state solution of Hirth and Lothe [11]. There have been two augmentations for these approaches: while Keralavarma et al. [12] developed a coarse-graining technique to incorporate diffusion of vacancies in determining the climb velocity of dislocations which enabled them to carry out DDP simulations over times on the order of hours, Ayas et al. [13] developed a methodology to solve the fully coupled dislocation motion and vacancy diffusion problem that demonstrates the importance of co-operative climb of dislocations with vacancies being exchanged by neighbouring climbing dislocations.

Moreover, the ability to solve boundary value problems using these DDP methodologies has more recently enabled Shishvan et al. [14] to predict the high temperature tensile response of two phase composites wherein dislocation motion in the matrix is via climb-assisted glide with the inclusions deforming by a combination of bulk elasticity and interfacial diffusion. Thus, in principle, the numerical tools to use DDP to predict the creep response of nickel-based superalloys including the effect of the inter-diffusion of the $\mathrm{Al}$ at the $\gamma / \gamma^{\prime}$ interface now exist.

Here, we present a DDP analysis of the onset of tertiary creep mode in nickel-based superalloys 
with the focus being on the role of the deformation at the $\gamma / \gamma^{\prime}$ interface via interfacial diffusion. The outline of the paper is as follows. First, we briefly describe the DDP framework of modelling superalloys including the effects of interfacial diffusion. Second, we discuss parameters used in the DDP calculations to represent the Ni superalloys and the temporal scaling of the DDP calculations in order to simulate deformation on the time scale of $1000 \mathrm{hrs}$. Finally, the DDP predictions are detailed with an emphasis on the mechanisms that govern the onset of tertiary creep regime in the superalloys.

\section{DDP modelling of superalloys}

DDP has been used extensively to analyse the deformation of composites comprising elastic particles in a single crystal matrix. In early studies such as the pioneering work of Cleveringa et al. [15], plastic deformation within the matrix was restricted to occur by only the glide motion of dislocations. More recently, Ayas et al. [16] extended this formulation such that matrix deformation is via both the glide and climb motion of dislocations and subsequently Shishvan et al. [14] incorporated the effect of deformation due to interfacial diffusion between the two phases of the composite. Here we shall employ the interfacial diffusion framework of Shishvan et al. [14] to investigate the creep response of nickel-based superalloys. However, unlike in both [16] and [14] where the dislocation climb velocity was modelled by a drag-type relation, we use the coupled framework of Ayas et al. [13] wherein climbing dislocations are treated as line sources/sinks of vacancies with the climb rate of dislocations dependent on the local vacancy concentrations. As demonstrated in [13] by ivestigating tension and bending of single crystals, the simpler formulation of drag-type relation slightly over-predicts the effect of dislocation climb, though the predictions are qualitatively similar. Therefore, it is preferred to employ the climb coupled with vacancy diffusion formulation [13].

We consider a two-dimensional (2D) plane-strain situation with deformation constrained in the $x_{1}-x_{2}$ plane. Consistent with the plane-strain condition, only straight edge dislocations perpendicular to the plane of deformation are considered (the dislocation lines are parallel to the $x_{3}$ axis). The 2D unit cell of nickel-based superalloy analysed here is sketched in Fig. 1a: the brickand-mortar micro-structure comprises a continuous Ni matrix $(\gamma)$ and identical rectangular $\mathrm{Ni}_{3} \mathrm{Al}$ 
precipitates $\left(\gamma^{\prime}\right)$. For the purposes of the present study, we neglect matrix/precipitate misfit stresses and the precipitates are taken to be coherent with the matrix. Deformation of the $\gamma$ phase is by a combination of glide and climb of dislocations while the $\gamma^{\prime}$ precipitates are assumed to undergo deformation by a combination of bulk elasticity and deformation due to inter-diffusion of the $\mathrm{Al}$ component of $\gamma^{\prime}$ phase within the lattice at the $\gamma / \gamma^{\prime}$ interface. We emphasise here that the unit cell in Fig. 1a serves as simple representation of the micro-structure of a nickel-based superalloy that captures the essential micro-structural features such as the blocking of the glide motion of dislocations in the $\gamma$ phase by the $\gamma^{\prime}$ precipitates. We shall now proceed to first explain the model for diffusion and deformation along the $\gamma / \gamma^{\prime}$ interfaces and then briefly describe overall DDP model; readers are referred to $[13,14]$ for further details.

\subsection{Diffusion along $\gamma / \gamma^{\prime}$ interfaces}

Spatial gradients in the normal stress along the interface between the $\gamma^{\prime}$ and $\gamma$ phases provides the driving force for inter-diffusion of the $\mathrm{Al}$ in the $\gamma^{\prime}$ phase along the interface and its consequent deformation, as sketched in Fig. 1b. We briefly outline the continuum approach to model this mass transport using the formulation introduced in [14].

The atomic chemical potential $\mu$ of the $\mathrm{Al}$ in the $\gamma^{\prime}$ phase along the $\gamma / \gamma^{\prime}$ interface is given in terms of the atomic volume $\Omega$ of $\mathrm{Al}$ in $\mathrm{Ni}_{3} \mathrm{Al}$ as [17]

$$
\mu=-\sigma_{\mathrm{n}} \Omega,
$$

where $\sigma_{\mathrm{n}}$ is the normal stress on the $\gamma / \gamma^{\prime}$ interface. Here, following Needleman and Rice [17], we have neglected the strain energy contribution to the chemical potential. Then, in the $2 \mathrm{D}$ context under consideration here, the volumetric flux $j(\xi)$ of Al along the $\gamma / \gamma^{\prime}$ interface is

$$
j(\xi)=\mathscr{D}_{(\mathrm{i})} \frac{\partial \sigma_{\mathrm{n}}(\xi)}{\partial \xi},
$$

where $\xi$ is a local co-ordinate along the $\gamma / \gamma^{\prime}$ interface as depicted in Fig. 1b. The interfacial diffusion constant $\mathscr{D}_{\text {(i) }}$ for $\mathrm{Al}$ within the Ni lattice through an interfacial zone of effective thickness $\delta_{\mathrm{b}}$ is related to the inter-diffusion coefficient $D_{\mathrm{b}}$ via

$$
\mathscr{D}_{(\mathrm{i})}=\frac{D_{\mathrm{b}} \delta_{\mathrm{b}} \Omega}{k T},
$$


where $k$ is the Boltzmann constant and $T$ is the absolute temperature. This mass transport results in the interpenetration of the $\gamma$ and $\gamma^{\prime}$ phases and can be expressed as a normal velocity discontinuity $\Delta v_{\mathrm{n}}$ between the $\gamma^{\prime}$ and $\gamma$ phases. Mass conservation then dictates $\Delta v_{\mathrm{n}}$ to be

$$
\Delta v_{\mathrm{n}}=-\frac{\partial}{\partial \xi}\left(\mathscr{D}_{(\mathrm{i})} \frac{\partial \sigma_{\mathrm{n}}(\xi)}{\partial \xi}\right)
$$

while we assume perfect bonding between the $\gamma$ and $\gamma^{\prime}$ phases such that there is no relative sliding at the $\gamma / \gamma^{\prime}$ interface. Moreover, mechanical equilibrium requires traction continuity across the $\gamma / \gamma^{\prime}$ interface and we enforce this via the solution of an appropriate DDP boundary value problem as described subsequently.

\subsection{The discrete dislocation plasticity model}

We consider a nickel-based superalloy subjected to uniaxial tension. Dislocation nucleation and motion takes place within the $\gamma$ phase on the slip systems oriented at angles $\varphi^{(1)}=\varphi$ and $\varphi^{(2)}=180^{\circ}-\varphi$ with respect to the $x_{1}$ axis and the $\gamma^{\prime}$ precipitates are assumed to be completely impenetrable to dislocations. Deformation of these $\gamma^{\prime}$ precipitates is by a combination of bulk elasticity and inter-diffusion of the $\mathrm{Al}$ as discussed in Section 2.1.

The periodic unit cell is assumed to be rectangular with dimensions $(h / \tan \varphi) \times h$ as shown in Fig. 1a. This choice of the aspect ratio ensures periodicity of the glide motion of the dislocations on discrete slip planes, i.e. a slip plane is available for dislocations exiting from one side of the unit cell to re-enter from the corresponding point on the opposite side of the unit cell. The rectangular $\gamma^{\prime}$ precipitates of size $w^{\gamma^{\prime}} \times h^{\gamma^{\prime}}$ are arranged in a regular cubic arrangement as shown in Fig. 1a and occupy a volume fraction $V_{\mathrm{f}}$ in the unit cell. Their aspect ratio $w^{\gamma^{\prime}} / h^{\gamma^{\prime}}$ is chosen such that for the given $V_{\mathrm{f}}$ the widths of the vertical and horizontal $\gamma$ channels are equal. The volume fraction $V_{\mathrm{f}}=0.7$ is used in all the calculations reported here and the above constraint then specifies the precipitate aspect ratio to be $w^{\gamma^{\prime}} / h^{\gamma^{\prime}} \simeq 0.84$. We note in passing that the arrangement of the $\gamma^{\prime}$ particles is not an issue in the present composite with narrow $\gamma$ channels and the arrangement shown in Fig. 1a was adopted following earlier DDP studies [15, 16]. Uniaxial tension with periodic boundary conditions is specified such that the difference in the displacement rates $\Delta \dot{u}_{i}$ of 
points separated by a position vector $\Delta x_{j}$ on opposite boundaries of the unit cell is

$$
\Delta \dot{u}_{i}=\dot{\bar{\varepsilon}}_{i j} \Delta x_{j}
$$

where $\dot{\bar{\varepsilon}}_{i j}$ is the macroscopic strain rate. Correspondingly, periodicity also dictates that the tractions $T_{i}$ at these corresponding locations on opposite boundaries of the unit cell are equal and opposite. The work-conjugate macroscopic stresses $\bar{\Sigma}_{i j}$ to $\dot{\bar{\varepsilon}}_{i j}$ follow from the boundary tractions as

$$
\bar{\Sigma}_{i j}=\frac{\tan \varphi}{2 h^{2}} \int_{C}\left(T_{i} x_{j}+T_{j} x_{i}\right) \mathrm{d} C,
$$

where $C$ is the boundary of the unit cell and uniaxial creep loading in the $x_{2}$ direction is imposed by requiring that $\bar{\Sigma}_{11}=\bar{\Sigma}_{12}=0$ while $\bar{\Sigma}_{22}$ is held fixed at the specified applied stress $\bar{\Sigma}^{\text {app }}$. Thus, in this formulation all strain rates $\dot{\bar{\varepsilon}}_{i j}$ are solution dependent variables with $\bar{\varepsilon}_{22}$ representing the creep strain under the applied stress $\bar{\Sigma}^{\text {app }}$. The specification of the boundary conditions is completed by imposing the normal velocity discontinuity, Eq. (4), at the $\gamma / \gamma^{\prime}$ interfaces along with traction continuity at these interfaces. Readers are referred to [14] for further details of the numerical procedures.

In DDP, the dislocations are treated as line defects in an otherwise elastic continuum with plasticity an outcome of the motion of these dislocations by a combination of glide and climb. While the long-range interactions of dislocations are captured by the linear elastic fields, the short-range interactions are incorporated through a set of constitutive rules introduced by Van der Giessen and Needleman [18] in the context of glide-only motion of dislocations and subsequently modified to include climb motion in $[10,13]$. These constitutive rules are governed by the glide and climb components of the Peach-Koehler $(\mathrm{P}-\mathrm{K})$ force on each dislocation $I$ denoted by $f_{\mathrm{g}}^{(I)}$ and $f_{\mathrm{c}}^{(I)}$, respectively. The glide velocity of dislocation $I$ is defined by a linear drag relation $v_{\mathrm{g}}^{(I)}=f_{\mathrm{g}}^{(I)} / B_{\mathrm{g}}$ where $B_{\mathrm{g}}$ is the drag coefficient. Computing the corresponding climb velocity $v_{\mathrm{c}}^{(I)}$ is more involved as it is dependent on the distribution of vacancies in the gamma phase. Here we follow the formulation introduced in [13] for the coupled high temperature vacancy diffusion and dislocation motion problem. For the sake of completeness, we briefly describe this formulation in the context of this superalloy problem. 
The climb velocity $v_{\mathrm{c}}^{(I)}$ of dislocation $I$ is set by the flux $\dot{N}_{\text {(v) }}$ of vacancies into the dislocation core such that mass conservation gives

$$
v_{\mathrm{c}}^{(I)}=-\frac{\Omega_{(\mathrm{v})} \dot{N}_{(\mathrm{v})}}{b^{(I)}}
$$

where $\Omega_{(\mathrm{v})}$ is the atomic vacancy volume and $b^{(I)}$ the signed magnitude of the Burgers vector of dislocation $I$. This vacancy flux in turn is given in terms of the climb P-K force and vacancy concentration $c\left(x_{i}^{(I)}\right)$ at the location of dislocation $I$ by

$$
\dot{N}_{(\mathrm{v})}=2 \pi r_{\mathrm{c}} K\left[c_{0} \exp \left(-\frac{f_{\mathrm{c}}^{(I)} \Omega_{(\mathrm{v})}}{b^{(I)} k T}\right)-c\left(x_{i}^{(I)}\right)\right],
$$

with $r_{\mathrm{c}}$ the so-called capture radius of the dislocation, $K$ a rate constant that governs the flux of vacancies across the dislocation core and $c_{0}$ the equilibrium concentration of vacancies at temperature $T$ in the absence of stress. It now remains to specify the vacancy concentration distribution $c\left(x_{i}\right)$. With $D_{\text {(v) }}$ denoting the diffusion constant of vacancies at temperature $T$, the spatio-temporal evolution of the vacancies follows a Fickian diffusion equation $\partial c / \partial t=D_{(\mathrm{v})} \nabla^{2} c$ subject to periodic boundary conditions over the boundaries of the unit cell and vacancies assumed to diffuse freely across the the $\gamma / \gamma^{\prime}$ interface. However, each climbing dislocation acts as a point source or sink of vacancies with a strength given by Eq. (8) and hence solving this diffusion equation is cumbersome. Either a coarse-graining technique [19] or a superposition principle [13] can be used to solve the governing diffusion equation. Here, we adopt the superposition principle introduced in [13] which involves employing the analytical solution for a point source in an infinite medium and then correcting for the boundary conditions. In this case of the periodic problem under consideration here, we correct for the boundary conditions by including images for each source/sink from eight neighbouring cells: numerical tests confirmed that these eight images were sufficient to resolve the vacancy fields to within $95 \%$ accuracy. It is the local vacancy concentration that determines the climb velocity of dislocations and the employed method to satisfy the periodic boundary conditions introduces very minor errors. However, note that each climbing dislocation leaves a trail of point vacancy sources and sinks. Thus, the number of vacancy sources/sinks increases rapidly in the calculation and including all of them in the numerical superposition becomes computationally prohibitive. In order to reduce the numerical cost, we delete a source/sink at a time $h^{2} /\left(4 D_{(v)}\right)$ after 
its nucleation: by this time the large gradients associated with the point source/sink have decayed and in fact the vacancy field associated with this source/sink is nearly uniform over the unit cell. Hence beyond this time we uniformly distribute the vacancy field of this source/sink over the unit cell by incrementing $c_{0}$ and no longer include it in the numerical superposition.

The material is assumed to be initially dislocation-free but dislocation sources with an areal density $\rho_{\text {nuc }}$ are randomly distributed through the $\gamma$ phase with equal numbers on both slip systems. These point sources are 2D representations of Frank-Read sources and nucleate a dipole of edge dislocations when the glide P-K force at the source exceeds the source strength $\tau_{\text {nuc }} b$ over a time period $t_{\text {nuc }}$, where $b$ is the magnitude of the Burgers vector. The Burgers vector of the nucleated dislocation is aligned with the slip system of the source and its sign is determined according to the direction of the glide P-K force at the source. Annihilation of two opposite signed dislocations on the same slip system occurs when they are sufficiently close together. Within the present framework, this is modeled by eliminating the dislocations when they are within a material-dependent critical annihilation distance $L_{\mathrm{e}}$. Unlike most DDP studies, we do not introduce any point obstacles to the dislocation motion in the $\gamma$ phase but the $\gamma^{\prime}$ precipitates are impenetrable to dislocations with the $\gamma / \gamma^{\prime}$ interfaces serving as large barriers to dislocation motion.

The stress and strain state are computed as a superposition of singular ( ) elastic dislocation fields and numerically computed smooth (^) fields that enforce the boundary conditions [18]. However, solving the interfacial diffusion equation and implementing the velocity discontinuity and traction continuity boundary conditions at the $\gamma / \gamma^{\prime}$ interface require a modification to the superposition scheme as detailed in Shishvan et al. [14] and here we employ this modified scheme and the associated numerical procedure. In brief, this involves the computation of the deformation history in an incremental manner due to the non-linearities introduced by the motion of the dislocations and the deformation due to interfacial diffusion. At each time increment this involves the following five main computational tasks:

(i) calculation of the glide and climb P-K forces on the dislocations from the known stress state at time $t$;

(ii) an update of the vacancy concentration field via the superposition of the fields of the active 
vacancy sources/sinks;

(iii) implementation of the short-range dislocation constitutive rules including the update of the dislocation positions via their glide and climb motion, their mutual annihilation and nucleation from sources;

(iv) calculation of the displacement jump at the $\gamma / \gamma^{\prime}$ interfaces; and

(v) determination of the stress and strain state at the next time increment $t+\Delta t$ by solving the complementary elastic finite element boundary value problem using the updated dislocation positions as well as the known boundary conditions on the $\gamma / \gamma^{\prime}$ interfaces and the exterior boundary of the unit cell.

\section{Temporal scaling of DDP calculations and material parameters}

Nickel-based superalloys display creep strain rates in the range $\dot{\varepsilon}^{\mathrm{c}}=10^{-9} \mathrm{~s}^{-1}-10^{-7} \mathrm{~s}^{-1}$ when subjected to typical uniaxial tensile stress of $\sim 300 \mathrm{MPa}$ at $\sim 950{ }^{\circ} \mathrm{C}[20,21,22]$. Such creep experiments are typically performed over about $1000 \mathrm{hrs}$ resulting in creep strains ranging from $0.4 \%$ to $40 \%$. DDP simulations typically require time-steps $\Delta t$ on the order of $0.5 \mathrm{~ns}$ so as to resolve short-range dislocation interactions such as dislocation nucleations, annihilations and pinning at the $\gamma / \gamma^{\prime}$ interfaces. Thus, it is impractical to directly perform creep DDP simulations over a time period of $1000 \mathrm{hrs}$ with temporally scaling the DDP simulations the more practical alternative.

Since deformation in these DDP simulations occurs by both dislocation climb (governed by vacancy diffusion) and interfacial diffusion, it is instructive to define non-dimensional time scales associated with both vacancy diffusion and interfacial diffusion while designing the temporal scaling of the DDP calculations. With $\ell$ denoting a representative dislocation spacing, time taken for vacancies to diffuse between co-operatively climbing dislocations (referred to as the vacancy diffusion time) is on the order $\ell^{2} / D_{\text {(v) }}$ while the loading time is on the order $1 / \dot{\varepsilon}^{\mathrm{c}}$. Thus, the ratio of the vacancy diffusion to loading time follows as

$$
\tau_{(\mathrm{v})}=\frac{\ell^{2} \dot{\varepsilon}^{\mathrm{c}}}{D_{(\mathrm{v})}} .
$$


Now consider the time for the $\mathrm{Al}$ to diffuse between dislocations near the interface (referred to as the interfacial diffusion time). Since the interfacial stresses are dependent on the local dislocation structure, they scale with the Young's modulus $E$ and thus a representative time for interfacial diffusion is $\ell^{3} /\left(\mathscr{D}_{(\mathrm{i})} E\right)$. Then, the ratio of the interfacial diffusion to loading time is

$$
\tau_{(\mathrm{i})}=\frac{\ell^{3} \dot{\varepsilon}^{\mathrm{c}}}{\mathscr{D}_{(\mathrm{i})} E} .
$$

In order to ensure that the dominant deformation mechanisms of the scaled DDP calculations are the same as in the creep experiments, we require that the non-dimensional time constants $\tau_{(\mathrm{v})}$ and $\tau_{(\mathrm{i})}$ are equal in the experiments and DDP calculations. Since we are only performing a temporal scaling, we shall use $\ell$ and $E$ values in the simulations appropriate for nickel-based superalloys. Thus, the scaling requirement reduces to $D_{(\mathrm{v})}^{\mathrm{EXP}} / D_{(\mathrm{v})}^{\mathrm{DDP}}=\dot{\varepsilon}^{\mathrm{DDP}} / \dot{\varepsilon}^{\mathrm{EXP}}$ and $\mathscr{D}_{(\mathrm{i})}^{\mathrm{EXP}} / \mathscr{D}_{(\mathrm{i})}^{\mathrm{DDP}}=\dot{\varepsilon}^{\mathrm{DDP}} / \dot{\varepsilon}^{\mathrm{EXP}}$, where the superscripts EXP and DDP denote the quantities in experiments on Ni superalloys and DDP simulations, respectively. Thus, $\lambda \equiv \dot{\varepsilon}^{\mathrm{DDP}} / \dot{\varepsilon}^{\mathrm{EXP}}$ represents a speed-up factor in the DDP simulations compared to the experiments. It should be emphasised that there is no any spatial scaling in the present model which ensures that the temporal scaling to be pursued as explained above. It follows that the time $t$ in the DDP calculations is related to time $t^{\mathrm{EXP}}$ in the experiments via $t^{\text {EXP }}=\lambda t$. All calculations presented subsequently employ a speed-up factor $\lambda=5 \times 10^{10}$. A discussion on the DDP calculations will be given in Section 4.3 in connection with results obtained.

\subsection{Reference parameters}

Scaling is only employed on parameters related to the diffusion properties of the nickel-based superalloys. We thus first detail the unscaled parameters and then explain the choice of the diffusion constants based on the temporal scaling described above. The $\gamma$ and $\gamma^{\prime}$ phases are assumed to be elastically identical and isotropic with $E=100 \mathrm{GPa}$ and $v=0.37$. Plasticity is constrained to the $\gamma$ phase which is initially dislocation-free but contains randomly distributed dislocation sources with density $\rho_{\text {nuc }}$ on planes spaced at a distance $s=100 \mathrm{~b}$ apart. The strength of dislocation sources is set by the Orowan stress to bow a dislocation loop through the $\gamma$ channels. Assuming a channel width of $90 \mathrm{~nm}$, this Orowan stress is $\sim 100 \mathrm{MPa}$ and thus the strength of each source is selected 
from a Gaussian distribution with a mean $\bar{\tau}_{\text {nuc }}=100 \mathrm{MPa}$ and a standard deviation of $2 \mathrm{MPa}$ while the nucleation time for all sources is taken as $t_{\text {nuc }}=10 \mathrm{~ns}$. Calculations with $\rho_{\text {nuc }}=300,500$ and $800 \mu \mathrm{m}^{-2}$ are reported (cf. Appendix A). These sources generate edge dislocation dipoles with a Burgers vector of magnitude $b=0.25 \mathrm{~nm}$ and the glide drag coefficient of these dislocations is $B_{\mathrm{g}}=10^{-4} \mathrm{~Pa}$.

The atomic volume of $\mathrm{Al}$ is $\Omega=16.6 \times 10^{-12} \mu \mathrm{m}^{3}$ while the $\gamma / \gamma^{\prime}$ interface thickness is taken to be $\delta_{\mathrm{b}}=1 \mathrm{~nm}$. The creep calculations are reported at a temperature $T=950{ }^{\circ} \mathrm{C}$ with an atomic vacancy volume $\Omega_{(\mathrm{v})}=13.8 \times 10^{-12} \mu \mathrm{m}^{3}$, an equilibrium vacancy concentration $c_{0}=20 \times 10^{6} \mu \mathrm{m}^{-3}$ and following Ayas et al. [13] we take the dislocation capture radius to be $r_{\mathrm{c}}=50 \mathrm{~b}$. The choice for the temperature at which the simulations are carried out is based on the experimental data (e.g. $[20,21]$ ) where this temperature was employed to investigate tertiary creep in commercially important nickel-based superalloys. It now remains to specify the scaled diffusion parameters $D_{(\mathrm{v})}^{\mathrm{DDP}}$ and $\mathscr{D}_{(\mathrm{i})}^{\mathrm{DDP}}$. At $T \simeq 1000{ }^{\circ} \mathrm{C}$, measurements suggest that $D_{(\mathrm{v})}^{\mathrm{EXP}} \approx 4 \times 10^{-6} \mu \mathrm{m}^{2} \mathrm{~s}^{-1}$ (see for example [3] ) and thus employing the scaling factor of $\lambda=5 \times 10^{10}$, we use the diffusion co-efficient $D_{\text {(v) }}^{\text {DDP }} \approx 2 \times 10^{5} \mu \mathrm{m}^{2} \mathrm{~s}^{-1}$ in the DDP calculations. However, there is more uncertainty with regards the inter-diffusion co-efficient of $\mathrm{Al}$ in Ni with measurements suggesting $D_{\mathrm{b}} \approx 10^{-7} \mu \mathrm{m}^{2} \mathrm{~s}^{-1}$ (see for example [23]). Calculations are presented for a reference value of $D_{\mathrm{b}}=10^{-7} \mu \mathrm{m}^{2} \mathrm{~s}^{-1}$ which gives the interfacial diffusion constant as $\mathscr{D}_{(\mathrm{i})}^{\mathrm{EXP}}=10^{-7} \mu \mathrm{m}^{5} \mathrm{~N}^{-1} \mathrm{~s}^{-1}$ (i.e. a scaled value of $\mathscr{D}_{(\mathrm{i})}^{\text {DDP }}=$ $5 \times 10^{3} \mu \mathrm{m}^{5} \mathrm{~N}^{-1} \mathrm{~s}^{-1}$ for the choice of $\lambda=5 \times 10^{10}$ ). However, to account for the uncertainty in the value of $D_{\mathrm{b}}$ we also present some parametric studies to illustrate the effect of interfacial diffusion. These studies are presented in terms of the non-dimensional group

$$
\overline{\mathscr{D}}=\frac{\mathscr{D}_{\text {(i) }} E}{D_{(\mathrm{v})} s},
$$

which is ratio of the vacancy diffusion to interfacial diffusion time. The reference values of the parameters correspond to $\overline{\mathscr{D}}=0.1$ and parametric studies are presented for $0 \leq \overline{\mathscr{D}} \leq 0.1$ with $\overline{\mathscr{D}}=$ 0 corresponding to the case when interfacial diffusion is absent. Finally, it remains to specify the rate constant $K$ that governs the vacancy kinetics across the dislocation core. The climb velocities of dislocations in the $\gamma$ phase of nickel-based superalloys are governed by the rate of vacancy diffusion rather than kinetics across the dislocation core. Thus, we take $D_{(\mathrm{v})} /\left(K r_{\mathrm{c}}\right)=0.15$ so that 
following Ayas et al. [13] the climb velocity of the dislocations is vacancy diffusion-limited with further increases in the value of $K$ not affecting the numerical results.

All calculations are presented for a nickel-based superalloy with a volume fraction $V_{\mathrm{f}}=0.7$ of $\gamma^{\prime}$ precipitates in a unit cell of size $h=0.6 \mu \mathrm{m}$, so that the $\gamma$ channels have a width of $90 \mathrm{~nm}$. The image fields in the DDP calculations were evaluated via finite element calculations wherein the unit cell was discretised with $0.01 \mu \mathrm{m}$ bi-linear quadrilaterals. Moreover, in order to account for the inevitable statistical effects in the DDP calculations we present results that are averages of three calculations with different realisation of the source locations and strengths.

\section{Results and discussion}

We now proceed to present numerical predictions for the creep response of nickel-based superalloys using the formulation and material properties detailed above. The creep calculations were performed in two steps. In step 1, a constant strain rate uniaxial tensile calculation was performed by applying strain rate of $0.4 \times 10^{-6} \mathrm{~s}^{-1}$ until the work-conjugate tensile stress $\bar{\Sigma}_{22}$ achieved the required value $\bar{\Sigma}^{\mathrm{app}}$ at a time $t^{\mathrm{EXP}}=t^{\mathrm{I}}$. Then in step 2, this stress $\bar{\Sigma}^{\mathrm{app}}$ was maintained constant for $t^{\mathrm{EXP}} \approx 1000$ hrs. We define $\bar{\varepsilon}^{\text {creep }}$ as the accumulated strain $\bar{\varepsilon}_{22}$ in step 2 and $t^{\text {creep }} \equiv t^{\mathrm{EXP}}-t^{\mathrm{I}}$ so that creep responses are presented for $\bar{\varepsilon}^{\text {creep }}$ as a function of $t^{\text {creep }}$ in line with typical experiments. Results are presented for two values of $\bar{\Sigma}^{\text {app }}$ specified as $\bar{\Sigma} \equiv \bar{\Sigma}^{\text {app }} / \bar{\tau}_{\text {nuc }}=3.8$ and 6.1 which are subsequently referred to as the low and high applied stress cases, respectively. It should be noted that the flow strength of these superalloys is predicted to be $(6 \sim 8) \times \bar{\tau}_{\text {nuc }}$, as evident from stress-strain curves presented in Appendix A.

\subsection{Low applied stress}

In this section we discuss the predictions of the creep response of the superalloys for the low applied normalised stress $\bar{\Sigma}=3.8$. DDP predictions of the creep response of the superalloys are plotted in Fig. 2 for the reference value of $\overline{\mathscr{D}}=0.1$ and $\overline{\mathscr{D}}=0$ corresponding to no interfacial diffusion and the three source densities considered here. In Fig. 2a, results are presented for the variation of the creep strain $\bar{\varepsilon}^{\text {creep }}$ with creep time $t^{\text {creep }}$ while in Fig. $2 \mathrm{~b}$ these results are replotted in terms of the temporal evolution of the creep strain rate $\dot{\bar{\varepsilon}}^{\text {creep }} \equiv \mathrm{d} \bar{\varepsilon}^{\text {creep }} / \mathrm{d} t^{\text {creep }}$. First consider 
the $\overline{\mathscr{D}}=0$ case. For low and intermediate source densities, the creep strain initially increases before plateauing out such that $\dot{\bar{\varepsilon}}^{\text {creep }} \approx 0$; see Fig. reffig:epscreep-time1b. The superalloy with the highest source density continues to creep even though over the time-scale investigated here the creep strain rates are low and remain approximately constant, i.e. the alloy remains in a secondary creep regime. Now consider the $\overline{\mathscr{D}}=0.1$ case. Now for all the source densities there is substantial creep of the alloys with $\dot{\bar{\varepsilon}}^{\text {creep }}$ increasing with increasing $\rho_{\text {nuc }}$. More importantly, for the two higher values of $\rho_{\text {nuc }}$, the creep responses of superalloys transition from a secondary creep regime to tertiary creep at $t^{\text {creep }} \approx 200 \mathrm{hrs}$ with $\dot{\bar{\varepsilon}}^{\text {creep }}$ increasing with increasing time for $t^{\text {creep }}>200 \mathrm{hrs}$. Tertiary creep has been extensively reported in the literature [4] for nickel-based superalloys and often the transition to tertiary creep is attributed to damage mechanisms such as void growth and interface damage; see for example [24, 25]. However, experimentally it is apparent that no significant damage (voids, cracks or recrystallization) occurs until strains in excess of $\sim 5 \%$ are accumulated [26]. For this reason such damage mechanisms are absent in our calculations and thus it is instructive to examine the source of the tertiary response predicted here.

The temporal evolution of the dislocation density $\rho_{\mathrm{dis}}$ in the $\gamma$ phase $\left(\rho_{\mathrm{dis}}\right.$ is defined as the ratio of the number of dislocations in the $\gamma$ phase to the area of the $\gamma$ phase) for the results in Fig. 2a is included in Fig. 2c. Clearly the rate of increase in $\rho_{\text {dis }}$ increases with increasing $\overline{\mathscr{D}}$ and $\rho_{\text {nuc }}$ and it is likely that the higher dislocation densities result in the higher creep strain rates seen in Fig. 2 b. There is one exception to this: for the highest source density and in the absence of interfacial diffusion, a steady-state creep behaviour is predicted while the dislocation density remains almost constant. Detailed investigations demonstrate that this behaviour can be rationalised by assuming that dislocation annihilation and multiplication are operating at rates which are balanced so that the dislocation density is constant. While such a steady-state creep behaviour is typically observed in pure nickel [4], the high source density used here for the superalloy has caused this behavior to occur.

In order to understand the effect of the higher dislocation densities, the dislocation structures for a single realisation of the $\rho_{\text {nuc }}=500 \mu \mathrm{m}^{2}$ superalloy are included in Figs. $3 \mathrm{a}$ and $3 \mathrm{~b}$ for $\overline{\mathscr{D}}=0$ and 0.1 , respectively at four selected values of $t^{\text {creep }}$. Early in the time history ( $t^{\text {creep }}=14 \mathrm{hrs}$ ) the dislocations are mainly concentrated at the $\gamma / \gamma^{\prime}$ interfaces for both the $\overline{\mathscr{D}}=0$ and 0.1 cases. 
With increasing time, a few dislocations are seen within the $\gamma$ phase in the $\overline{\mathscr{D}}=0$ while the dislocation densities are larger in the $\overline{\mathscr{D}}=0.1$ case with dislocation climb enabling the formation of low energy wall structures. In fact, around $t^{\text {creep }}=900 \mathrm{hrs,} \mathrm{high} \mathrm{dislocation} \mathrm{densities} \mathrm{and}$ dislocation cell structures are evident in the $\overline{\mathscr{D}}=0.1$ case consistent with numerous experimental observations; see for example Pollock and Argon [27] and more recently [28, 29]. On the other hand, very limited dislocation activity is observed within the $\gamma$ phase (with nearly all dislocations restricted to the $\gamma / \gamma^{\prime}$ interface) in the $\overline{\mathscr{D}}=0$ case. We shall proceed to argue that this is a result of the fact that in a constant applied stress creep test, dislocation climb and vacancy diffusion cease early in the deformation history with $\overline{\mathscr{D}}=0$ as back stresses of the dislocations pilep-up at the $\gamma / \gamma^{\prime}$ interface inhibit further dislocation nucleation. By contrast, when interfacial diffusion is active, the $\gamma / \gamma^{\prime}$ interface undergoes a diffusion-assisted deformation allowing dislocations at the interface to continue to climb. This relaxes the local stresses and since the applied stress is higher than the flow strength of the matrix (cf. Appendix A) it enables additional dislocation nucleation and the development of the dislocation cell structure. This additional dislocation activity in the $\gamma$ phase is what results in the increasing creep strain rate (tertiary creep) seen in Fig. 2b. We now proceed to interrogate the numerical results to demonstrate this hypothesis.

The spatial distributions of the normalised vacancy concentration $c / c_{0}$ are included in Figs. $4 \mathrm{a}$ and $4 \mathrm{~b}$ for a single realisation of the $\rho_{\text {nuc }}=500 \mu \mathrm{m}^{-2}$ superalloy with $\overline{\mathscr{D}}=0$ and 0.1 , respectively. These distributions at four selected values of $t^{\text {creep }}$ indicate that with $\overline{\mathscr{D}}=0$ there are spatial gradient in $c$ for $t^{\text {creep }} \leq 300 \mathrm{hrs}$ with the vacancy distribution being approximately spatially uniform thereafter. By contrast, spatial gradients in $c$ persist in the $\overline{\mathscr{D}}=0.1$ case over the entire time history computed here. Dislocation climb in the $\gamma$ phase occurs by the co-operative motion of dislocations as discussed in [13], i.e. dislocations or groups of dislocations climb by emitting vacancies while another set in their vicinity climb by absorbing vacancies. Thus, the spatial gradients in $c$ are indicative that deformation by dislocation climb continues in the $\overline{\mathscr{D}}=0.1$ case throughout the time history computed here while, with $\overline{\mathscr{D}}=0$, dislocation climb ceases very early in the time history. To illustrate the deformation due to dislocation climb in the $\gamma$ phase we define an average strain 
measure

$$
\bar{\epsilon}_{\mathrm{c}}=\frac{1}{A^{\gamma}} \int_{A^{\gamma}} \sum_{\alpha=1}^{2}\left|m_{i}^{(\alpha)} E_{i j} m_{j}^{(\alpha)}\right| \mathrm{d} A,
$$

where $A^{\gamma}$ is the area of the $\gamma$ phase, $m_{i}^{(\alpha)}$ the unit normal of slip system $(\alpha)$ and $E_{i j}=0.5\left(u_{i, j}+\right.$ $u_{j, i}$ ) the strain calculated by numerically differentiating the total displacement field $u_{i}$ using the FE mesh comprising square bi-linear elements of size $0.01 \mu \mathrm{m}$. These strains are an order of magnitude higher than the creep strains in Fig. 2, because deformation of the superalloy is mainly due to deformation within the $\gamma$ channels. Predictions of the temporal evolution of $\bar{\epsilon}_{\mathrm{c}}$ are included in Fig. 5 for both $\overline{\mathscr{D}}=0$ and 0.1 and the three source densities considered here. It is clear that not only are the accumulated strains due to dislocation climb in the $\gamma$ phase larger in the $\overline{\mathscr{D}}=0.1$ case but more importantly $\dot{\bar{\epsilon}}_{\mathrm{c}}$ increases with increasing time as demonstrated only in the $\overline{\mathscr{D}}=0.1$ case. This reveals that the increase in creep rate of the superalloy is due to an increasing deformation rate of the $\gamma$ phase due to dislocation climb ${ }^{1}$. We emphasise that the strain-like measure (12) cannot differentiate between extra plastic strain due to enhanced climb of each dislocation and increased plastic strain due to a larger number of climbing/gliding dislocations being present. While we expect that the higher $\bar{\epsilon}_{\mathrm{c}}$ in the $\overline{\mathscr{D}}=0.1$ case is mainly due to more dislocations being generated, the DDP calculations cannot directly confirm this.

In fact, unlike in continuum plasticity predictions [30] of the deformation of composites in the presence of interfacial diffusion, the elongation of the $\gamma^{\prime}$ precipitates due to interfacial diffusion has only a small contribution to the increasing creep strain of the superalloy. To illustrate this we define a measure of average strain within the $\gamma^{\prime}$ precipitate

$$
\bar{\varepsilon}_{i j}^{\gamma^{\prime}}=\frac{1}{2 A^{\gamma^{\prime}}} \oint_{C^{\gamma^{\prime}}}\left(\mathscr{U}_{i}^{\gamma^{\prime}} n_{j}^{\gamma^{\prime}}+\mathscr{U}_{j}^{\gamma^{\prime}} n_{i}^{\gamma^{\prime}}\right) \mathrm{d} C
$$

where $A^{\gamma^{\prime}}$ and $C^{\gamma^{\prime}}$ are the area and perimeter, respectively, of the precipitate, $\mathscr{U}_{i}^{\gamma^{\prime}}$ is the total displacement of the precipitate surface (due to both bulk elastic deformation and interfacial diffusion)

\footnotetext{
${ }^{1}$ We note in passing that while this extensive plastic deformation due to dislocation climb is a result of vacancy diffusion, in these DDP simulations there is no vacancy accumulation resulting in void formation. Such void induced damage mechanisms have been invoked in the literature [24] to rationalise the use of damage based models for the tertiary creep response at strains in excess of $\sim 5 \%$.
} 
and $n_{i}^{\gamma^{\prime}}$ the outward normal to the surface of the precipitate; see Shishvan et al. [14] for further details of this deformation measure. Predictions of $\bar{\varepsilon}_{22}^{\gamma^{\prime}}$ as a function of $t^{\text {creep }}$ for the central precipitate of the unit cell are included in Fig. 6a for both $\overline{\mathscr{D}}=0$ and $\overline{\mathscr{D}}=0.1$ and the three source densities considered here. In the absence of interfacial diffusion $(\overline{\mathscr{D}}=0)$, the elongation of the $\gamma^{\prime}$ precipitate is negligible but even with interfacial diffusion present $(\overline{\mathscr{D}}=0.1)$ the contribution of the precipitate deformation to the overall deformation is small; compare Figs. 6a and 2a. Moreover, the precipitate strain increases linearly with time while the deformation of the superalloy accelerates in the $\overline{\mathscr{D}}=0.1$ case. This confirms that the increasing creep strain rate of the superalloy in the $\overline{\mathscr{D}}=0.1$ case is largely due to the acceleration of deformation due to dislocation climb within the $\gamma$ phase.

While interfacial diffusion does not directly contribute significantly to the deformation of the superalloy, it is clear that it plays a major indirect role as demonstrated from differences in the overall creep responses in the $\overline{\mathscr{D}}=0$ and 0.1 cases. The deformed shapes of the central precipitate are illustrated in Fig. 3 (with the deformations magnified by a factor of 6 to aid visualisation). Negligible deformations are observed in the $\overline{\mathscr{D}}=0$ case and consistent with the results in Fig. 6a, the overall deformations are relatively small even with $\overline{\mathscr{D}}=0.1$. However, large local deformations are observed when $\overline{\mathscr{D}}=0.1$. To quantify these local deformations, we define a precipitate surface displacement $\Delta u_{\mathrm{n}}=\int \Delta v_{\mathrm{n}} \mathrm{d} t$ and include in Fig. 6b predictions of the temporal evolution of $\Delta u_{\mathrm{n}} / b$ at four locations on the surface of the central precipitate as indicated in the inset. These results are shown for a single realisation of the $\rho_{\text {nuc }}=500 \mu \mathrm{m}^{-2}$ superalloy with $\overline{\mathscr{D}}=0.1$ (i.e. the case in Fig. 3b). Interfacial diffusion is seen to be able to cause surface perturbations in the $\gamma^{\prime}$ precipitates on the order of $10 b-20 b$ (or $\sim 5 \%$ of the $\gamma$ phase channel size). Indeed, waviness of the $\gamma / \gamma^{\prime}$ interfaces of this order has been experimentally observed in nickel-based superalloys subjected to creep deformation at temperatures below which rafting occurs; see for example [5, 6, 31, 32]. Indeed, Vorontsov et al. [6] argue that the serration of the interface is a result of inter-diffusion of the $\mathrm{Al}$ in the Ni driven by the elastic strain energy of the dislocations near the $\gamma / \gamma^{\prime}$ interface: the DDP calculations presented here capture this mechanism. These local deformations of the interface relax the stresses within the $\gamma$ channels allowing for continued nucleation of dislocations similar to that reported by Danas et al. [33] in the context of a single crystal sheared between 
compliant (elastic) platens. The continued nucleation of dislocations results in a high dislocation density (cf. Fig. 2c) with dislocation climb enabling the arrangement of these dislocations first into low energy wall structures and then cell structures with increasing dislocation density. The cell walls provide pathways for easy dislocation climb and we attribute the increasing creep strain rate to this dislocation structure formation in the $\gamma$ phase.

\subsubsection{Sensitivity of the creep response to the interfacial diffusion constant $\overline{\mathscr{D}}$}

Interfacial diffusion is seen to have a significant effect on the creep response of the superalloys. While $\overline{\mathscr{D}}=0.1$ is a realistic value for current materials it is conceivable that interface engineering can enable $\overline{\mathscr{D}}$ to be reduced. With this in mind we present here DDP predictions of the sensitivity of the creep response to $\overline{\mathscr{D}}$. Predictions of $\bar{\varepsilon}^{\text {creep }}$ and the corresponding $\dot{\bar{\varepsilon}}^{\text {creep }}$ versus creep time $t^{\text {creep }}$ are included in Figs. 7a and 7b, respectively for the $\rho_{\text {nuc }}=500 \mu \mathrm{m}^{-2}$ superalloy and selected values of $\overline{\mathscr{D}}$ in the range $0 \leq \overline{\mathscr{D}} \leq 0.1$. With decreasing $\overline{\mathscr{D}}$, not only does the creep strain rate decrease but importantly, for $\overline{\mathscr{D}} \leq 0.01$, tertiary creep regime is precluded over the time-scales investigated here. This suggests that in addition to reducing the diffusion rates in the $\gamma$ matrix to inhibit dislocation climb (for example with Re additions [34]), reducing interfacial diffusion via slow-diffusing solutes in the $\gamma^{\prime}$ phase could also enable significant improvements to the creep response of superalloys.

\subsection{High applied stress}

All results presented so far are for the applied normalised stress $\bar{\Sigma}=3.8$ and suggest that the creep strain rates are very low in the absence of interfacial diffusion. We proceed now to investigate the effect of applying a higher normalised stress level $\bar{\Sigma}=6.1$ with the creep strain $\bar{\varepsilon}^{\text {creep }}$ versus creep time $t^{\text {creep }}$ curves included in Fig. 8a for both the $\overline{\mathscr{D}}=0$ and 0.1 cases and the three source densities considered in this study. The creep rates are now much higher compared with the $\bar{\Sigma}=3.8$ case and in fact the superalloy continues to creep over the entire time-scale investigated here even with $\overline{\mathscr{D}}=0$; compare Figs. 2a and 8a. This creep is due to deformation of the $\gamma$ phase by dislocation climb as seen in Fig. 8b which shows the temporal evolution of $\bar{\epsilon}_{\mathrm{c}}$ for the cases included in Fig. 8a. Now unlike for the low applied stress case (cf. Fig. 5), the average strain due to dislocation climb in the $\gamma$ phase continues to increase with time even when $\overline{\mathscr{D}}=0$. 
In the $\bar{\Sigma}=3.8$ case with the absence of interfacial diffusion, dislocations are primarily present at the $\gamma / \gamma^{\prime}$ interface and we argue that the back-stresses of these dislocations inhibit continued dislocation nucleation and creep deformation of the $\gamma$ phase. To understand how this changes for the higher applied stress level, we include in Fig. 9 the dislocation structures in the $\rho_{\text {nuc }}=500 \mu \mathrm{m}^{-2}$ superalloy subjected to $\bar{\Sigma}=6.1$ at three selected times $t^{\text {creep }}$ for the no interfacial diffusion case $(\overline{\mathscr{D}}=0)$. Very early in the deformation history $\left(t^{\text {reep }}=14 \mathrm{hrs}\right)$, similar to Fig. $3 \mathrm{a}$, we see dislocations piled-up against the $\gamma / \gamma^{\prime}$ interface. However, unlike in Fig. 3a, dislocations are also observed throughout $\gamma$ phase. These dislocations are able to move by a combination of glide and climb resulting in deformation of the $\gamma$ phase and consequently also the superalloy. Moreover, the evolving dislocation structure implies the stresses on the dislocation sources also evolve resulting in further dislocation nucleation. In fact, a relatively high dislocation density develops within the $\gamma$ phase by $t^{\text {creep }}=700 \mathrm{hrs}$ and dislocation climb enables these dislocations to arrange themselves into low energy cell structures. Thus, unlike with $\bar{\Sigma}=3.8$, now dislocation cell structures develop with $\bar{\Sigma}=6.1$ even in the absence of interfacial diffusion and these cell structures increase the creep strain rate of the superalloy. We note in passing that the deformations of the central precipitate shown in Fig. 9 are solely a result of bulk elastic deformations at this high applied stress level.

Thus, while a reduction in the interfacial diffusion constant reduces or even switches off creep at low applied stress levels, dislocation climb and associated creep occurs for higher applied stress levels even in the absence of interfacial diffusion. The DDP calculations thus predict that interfacial diffusion serves to increase creep strain rates at any given applied stress due to deformations of the $\gamma / \gamma^{\prime}$ interface.

\subsection{Discussion on DDP calculations}

The results presented here for creep of superalloys have been obtained via the climb-ebabled DDP framework developed in [13] to consider coupling of vacancy diffusion and dislocation climb. This framework has certain similarities to the formulation developed in $[12,19]$ for hightemperature DDP. To be specific, the climb velocity of dislocations is calculated through the expressions (7)-(8) in both formulations. However, while the framework of Ayas et al. [13] captures the co-operative motion of dislocations as it containes the full dynamics of discrete dislo- 
cations (i.e. climb and glide) in a single time-frame, the formulation of Keralavarma and Benzerga [19] employs a staggered solution methodology in two time-frames with application of a coarse-graining technique. Because of significant differences between glide and climb velocities of dislocations, performing simulations via framework in [13] using "real" vacancy diffusion constant becomes unfeasable whereas the formulation in [19] makes it come true with the fact that the co-operative motion of dislocations is overlooked.

Now, for the present problem of creep in superalloys, glide motion of dilocations is very constrained by the $\gamma^{\prime}$ particles and therefore glide velocities are very small. Subsequently, the motion of dislocations is climb dominated. This is the key in applying temporal scaling of DDP calculations as explained in Section 3 where negligible glide motion of dislocations and associated time do not involve in defining the speed-up factor. It is however noted that the formation of cell structures, as predicted in the superalloys, requires the climb and (very small) glide motion of dilocations to occur simultaneously in a single time-frame.

\section{Concluding remarks}

The high temperature creep response of single crystal nickel-based superalloys with a brickand-mortar microstructure comprising $\mathrm{Ni}_{3} \mathrm{Al}$ precipitates $\left(\gamma^{\prime}\right)$ in a $\mathrm{Ni}$ matrix $(\gamma)$ has been investigated using discrete dislocation plasticity (DDP). The $\gamma^{\prime}$ precipitates are elastic but also undergo deformation due to stress-driven inter-diffusion of $\mathrm{Al}$ in Ni lattice at the $\gamma / \gamma^{\prime}$ interface while plastic deformation of the $\gamma$ phase is by a combination of dislocation glide and climb driven by vacancy diffusion. The coupled dislocation motion, vacancy diffusion and interfacial diffusion problem is solved using the DDP formulations developed in Ayas et al. [13] and Shishvan et al. [14]. Creep simulations are presented at a temperature $\sim 950{ }^{\circ} \mathrm{C}$ with the alloy subjected to uniaxial tension.

For a relatively low applied stress, the creep rates are very low in the absence of interfacial diffusion: dislocations nucleated by the applied stress get pinned at the $\gamma / \gamma^{\prime}$ interface and the back stresses exerted by these dislocations inhibit further dislocation nucleation and thereby preclude continued plastic deformation of the $\gamma$ phase. By contrast, when interfacial diffusion is permitted, a serrated or wavy $\gamma / \gamma^{\prime}$ interface develops due to the inter-diffusion of $\mathrm{Al}$ in $\mathrm{Ni}$ lattice driven by the inhomogeneous stress fields of the dislocation structures in the $\gamma$ phase. This deformation 
at the interface relaxes the dislocation stresses and permits continued nucleation of dislocations. Dislocation climb allows for the high density of dislocations that now exist in the $\gamma$ phase to arrange themselves into low energy cell structures which further enhances the creep strain rate. In fact, the formation of the cell structures within the $\gamma$ phase results in the onset of the tertiary creep regime whence the creep strain rate of the superalloy increases with increasing time. Thus, unlike continuum plasticity predictions [30], the DDP simulations suggest that the contribution of the average deformation of the $\gamma^{\prime}$ precipitates to the overall strain of the superalloy is small. Rather the main effect of interfacial diffusion is indirect in that it allows for enhanced plasticity and cell structure formation within the $\gamma$ phase. At high applied stress levels, creep deformation of the superalloy is predicted even in the absence of interfacial diffusion. Now, dislocations are present throughout the $\gamma$ phase and not just pinned at the $\gamma / \gamma^{\prime}$ interfaces. These dislocations can continue to climb resulting in continued creep of the $\gamma$ phase and thereby also the superalloy. These creep rates are of course further enhanced when interfacial diffusion is active.

Recent experimental observations [5,6] have reported the formation of serrated or wavy $\gamma / \gamma^{\prime}$ interfaces during the tertiary creep deformation of nickel-based superalloys. The DDP analysis presented here not only predicts such deformations but importantly suggests that interfacial diffusion and the consequent interface deformations play a crucial role in setting the creep rates of nickel-based superalloys.

\section{Acknowledgments}

Support from ONR under grant numbers N62909-14-1N242 and N00014-14-1-0618 on Multiscale methods for creep resistant alloys (program manager Dr. David Shifler) is gratefully acknowledged. SSS/VSD would like to thank Dr. Can Ayas for useful discussions.

\section{Appendix A. Uniaxial stress-strain responses}

Figure 10 shows the uniaxial stress-strain curves predicted by DDP for the superalloy subjected to constant strain reate of $0.4 \times 10^{-6} s^{-1}$. It is illustrated that the flow strength of superalloys is $(6 \sim$ 8) $\times \bar{\tau}_{\text {nuc }}$ dependent on the dislocation source density and the interfacial diffusion constant. This 
is significantly higher than the matix flow strength (the same as it's low yield stress due to lack of hardening in pure $\gamma$ phase) which is estimated to be approximately $2 \times \bar{\tau}_{\text {nuc }}$ by using a Schmid factor of $0.5 \sin 2 \varphi$. Moreover, it should be emphasised that the values of source densities employed in the simulations are chosen based on the fact that, considering the value of $\bar{\tau}_{\text {nuc }}=100 \mathrm{MPa}$ used in simulations, the flow strength level of model superallys is to be similar to strength of commertially important nickel-based superalloys [4].

\section{References}

[1] T. Pollock, S. Tin, Nickel-Based Superalloys for Advanced Turbine Engines: Chemistry, Microstructure and Properties, J Propulsion and Power 22 (2006) 361-374.

[2] R. C. Reed, T. Tao, N. Warnken, Alloys-By-Design: Application to nickel-based single crystal superalloys, Acta Mater 57 (2009) 5898-5913.

[3] Z. Zhu, H. Basoalto, N. Warnken, R. C. Reed, A model for the creep deformation behaviour of nickel-based single crystal superalloys, Acta Mater 60 (2012) 4888-4900.

[4] R. C. Reed, The superalloys: fundamentals and applications, Cambridge press, cambridge, 2006.

[5] A. B. Parsa, P. Wollgramm, H. Buck, C. Somsen, A. Kostka, I. Povstugar, P. Choi, D. Raabe, A. Dlouhy, J. Mller, E. Spiecker, K. Demtroder, J. Schreuer, K. Neuking, G. Eggeler, Advanced Scale Bridging Microstructure Analysis of Single Crystal Ni-Base Superalloys, Adv Eng Mater 17 (2015) 216-230.

[6] V. A. Vorontsov, L. Kovarik, M. J. Mills, C. M. F. Rae, High-resolution electron microscopy of dislocation ribbons in a CMSX-4 superalloy single crystal, Acta Mater 60 (2012) 4866 - 4878.

[7] D. Raabe, On the consideration of climb in discrete dislocation dynamics, Phil Mag A 77 (1998) 751-759.

[8] S. M. Hafez Haghighat, G. Eggeler, D. Raabe, Effect of climb on dislocation mechanisms and creep rates in $\gamma^{\prime}$-strengthened Ni base superalloy single crystals: A discrete dislocation dynamics study, Acta Mater 61 (2013) 3709-3723.

[9] K. M. Davoudi, L. Nicola, J. J. Vlassak, Dislocation climb in two-dimensional discrete dislocation dynamics, J Appl Phys 111 (2012) 103522-1-7.

[10] K. Danas, V. S. Deshpande, Plane-strain discrete dislocation plasticity with climb-assisted glide motion of dislocations, Modell Simul Mater Sci Eng 21 (2013) 045008(26pp).

[11] J. P. Hirth, J. Lothe, Theory of Dislocations, Wiley, New York, 2 edn., 1982.

[12] S. M. Keralavarma, T. Cagin, A. Arsenlis, A. A. Benzerga, Power-law creep from discrete dislocation dynamics, Phys Rev Lett 109 (2012) 265504.

[13] C. Ayas, J. A. W. van Dommelen, V. S. Deshpande, Climb-enabled discrete dislocation plasticity, J Mech Phys Solids 62 (2014) 113-136. 
[14] S. S. Shishvan, T. M. Pollock, R. M. McMeeking, V. S. Deshpande, Interfacial diffusion in high-temperature deformation of composites: a discrete dislocation plasticity investigation, J Mech Phys Solids 98 (2017) 330349.

[15] H. H. M. Cleveringa, E. Van der Giessen, A. Needleman, Comparison of discrete dislocation and continuum plasticity predictions for a composite material, Acta Mater 45 (1997) 3163-3179.

[16] C. Ayas, L. C. P. Dautzenberg, M. G. D. Geers, V. S. Deshpande, Climb-enabled discrete dislocation plasticity analysis of the deformation of a particle reinforced composte, J Appl Mech 82 (2015) 071007-1-13.

[17] A. Needleman, J. R. Rice, Plastic creep flow effects in the diffusive cavitation of grain boundaries, Acta Mettal 28 (1980) 1315-1332.

[18] E. Van der Giessen, A. Needleman, Discrete dislocation plasticity: a simple planar model, Modell Simul Mater Sci Eng 3 (1995) 689-735.

[19] S. M. Keralavarma, A. A. Benzerga, High-temperature discrete dislocation plasticity, J Mech Phys Solids 82 (2015) 1-22.

[20] N. Matan, D. C. Cox, P. Carter, M. A. Rist, C. M. F. Rae, R. C. Reed, Creep of CMSX-4 superalloy single crystals: effects of misorientation and temperature, Acta Mater 47 (1999) 1549-1563.

[21] R. C. Reed, N. Matan, D. C. Cox, M. A. Rist, C. M. F. Rae, Creep of CMSX-4 superalloy single crystals: effects of rafting at high temperature, Acta Mater 47 (1999) 3367-3381.

[22] Y. Koizumi, T. Kobayashi, T. Yokokawa, J. Zhang, M. Osawa, H. Harada, Y. Aoki, M. Arai, Development of next-generation Ni-base single crystal superalloys, in: Superalloys, TMS, Warrendale, PA, USA, 35-43, 2004.

[23] T. Ikeda, A. Almazouzi, H. Numakura, M. Koiwa, W. Sprengel, H. Nakajima, Single-phase interdiffusion in $\mathrm{Ni}_{3} \mathrm{Al}$, Acta Metall Mater 46 (1998) 5369-5376.

[24] A. Ma, D. Dye, R. C. Reed, A model for the creep deformation behaviour of single-crystal superalloy CMSX-4, Acta Mater 56 (2008) 1657-1670.

[25] A. Staroselsky, B. Cassenti, Mechanisms for tertiary creep of single crystal superalloy, Mech Time-Depend Mater 12 (2008) 275-289.

[26] L. H. Rettberg, M. Tsunekane, T. Pollock, Rejuvenation of nickel-based superalloys GTD444(DS) and RENE N5(SX), Superalloys (2012) 35-43.

[27] T. M. Pollock, A. S. Argon, Creep resistance of CMSX-3 nickel base superalloy single crystals, Acta Metall Mater 40 (1992) 1-30.

[28] L. Agudo Jacome, P. Nortershauser, J.-K. Heyer, A. Lahni, J. Frenzel, A. Dlouhy, C. Somsen, G. Eggeler, Hightemperature and low-stress creep anisotropy of single-crystal superalloys, Acta Mater 61 (2013) 2926-2943.

[29] L. Agudo Jacome, P. Nortershauser, C. Somsen, A. Dlouhy, G. Eggeler, On the nature of phase cutting and its effect on high temperature and low stress creep anisotropy of Ni-base single crystal superalloys, Acta Mater 69 (2014) 246-264. 
[30] P. Sofronis, R. M. McMeeking, The effect of interface diffusion and slip on the creep resistance of particulate composite materials, Mech Mater 18 (1994) 55-68.

[31] T. Link, A. Epishin, M. Paulisch, T. May, Topography of semicoherent $\gamma / \gamma^{\prime}$-interfaces in superalloys: Investigation of the formation mechanism, Mater Sci Eng A 528 (2011) 6225-6234.

[32] A. B. Parsa, P. Wollgramm, H. Buck, A. Kostka, C. Somsen, A. Dlouhy, G. Eggeler, Ledges and grooves at $\gamma / \gamma^{\prime}$ interfaces of single crystal superalloys, Acta Mater 90 (2015) 105-117.

[33] K. Danas, V. S. Deshpande, N. A. Fleck, Compliant interfaces: A mechanism for relaxation of dislocation pile-ups in a sheared single crystal, Int J Plast 26 (2010) 1792-1805.

[34] A. Mottura, R. C. Reed, What is the role of rhenium in single crystal superalloys?, MATEC Web of CONFERENCES 14 (2014) 01001. 


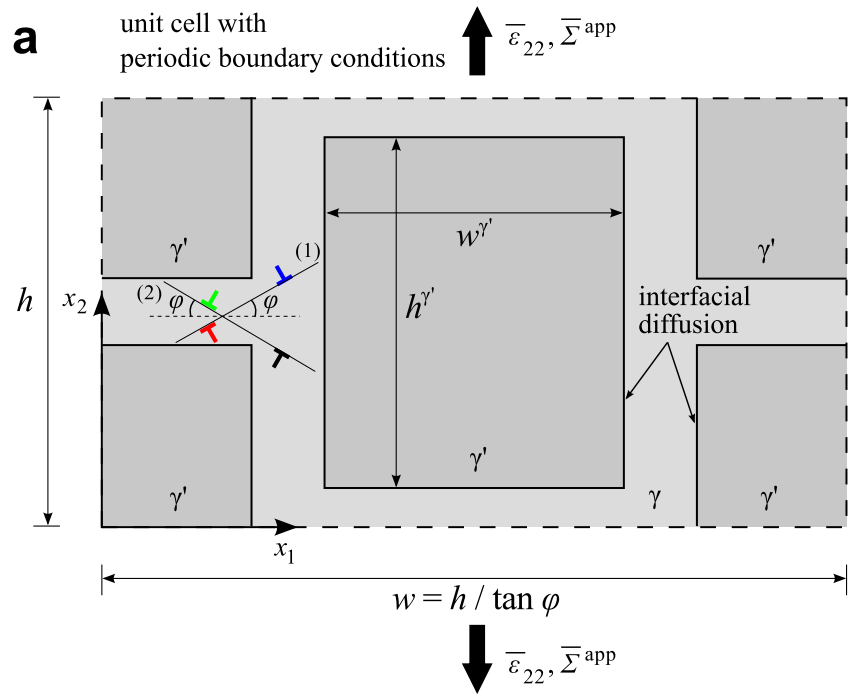

b

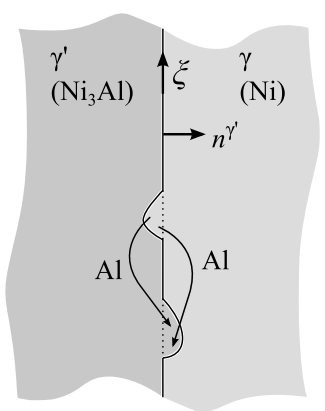

Figure 1: (a) Sketch of the unit cell of the superalloy with the brick-and-mortar microstructure analysed in this study. The rectangular unit cell subjected to uniaxial tensile loading in the $x_{2}$ direction comprises rectangular elastic $\gamma^{\prime}$ precipitates in an elastic-plastic $\gamma$ matrix phase. The $\gamma$ phase is a single crystal with two active slip systems as indicated: the colours used to depict the edge dislocations on these slip systems are utilised to illustrate the dislocation structures in the remainder of the paper. (b) A schematic of inter-diffusion of $\mathrm{Al}$ in $\mathrm{Ni}$ along the $\gamma / \gamma^{\prime}$ interface with $\xi$ the local co-ordinate along the interface and $n^{\gamma^{\prime}}$ the unit outward normal to the $\gamma^{\prime}$ precipitate. 

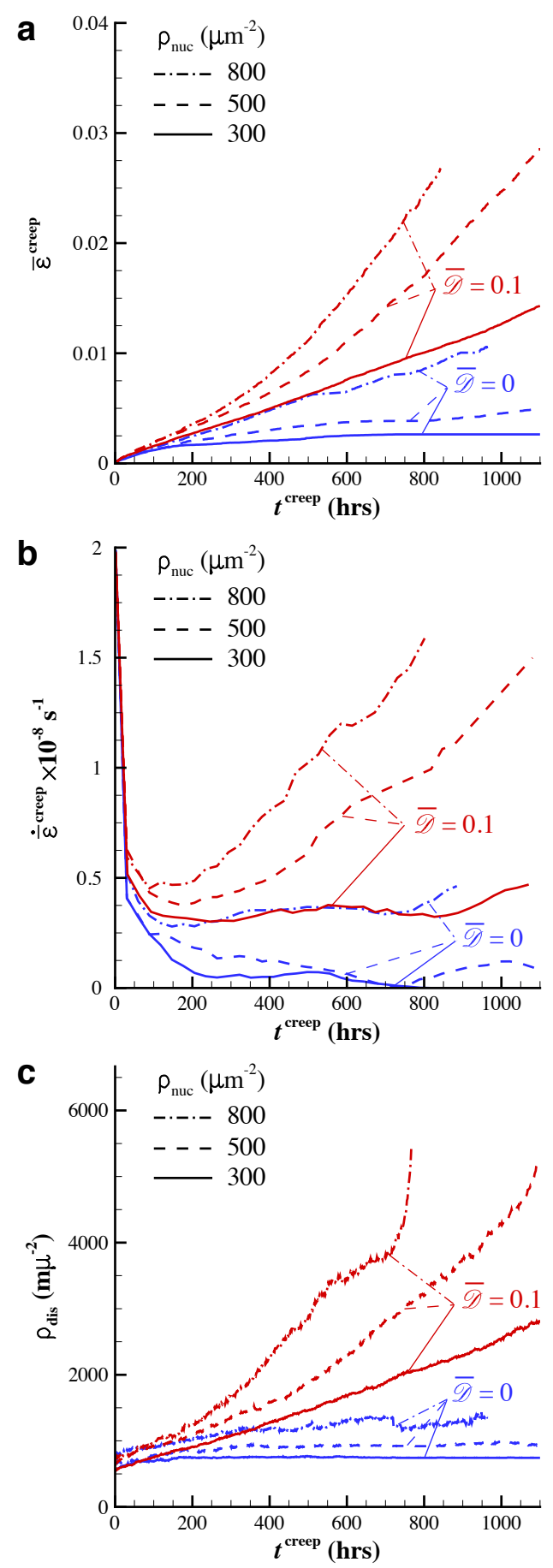

Figure 2: DDP predictions of (a) the creep strain $\bar{\varepsilon}^{\text {creep }}$ versus the creep time $t^{\text {creep }}$ and the corresponding temporal evolution of (b) the creep strain rate $\dot{\bar{\varepsilon}}^{\text {creep }}$ and (c) the dislocation density $\rho_{\text {dis }}$ within the $\gamma$ phase. Results are shown for an applied normalised stress $\bar{\Sigma}=3.8$ for superalloys with normalised interfacial diffusion constants $\overline{\mathscr{D}}=0$ and 0.1 and three values of density $\rho_{\text {nuc }}$ of dislocation sources. 
a
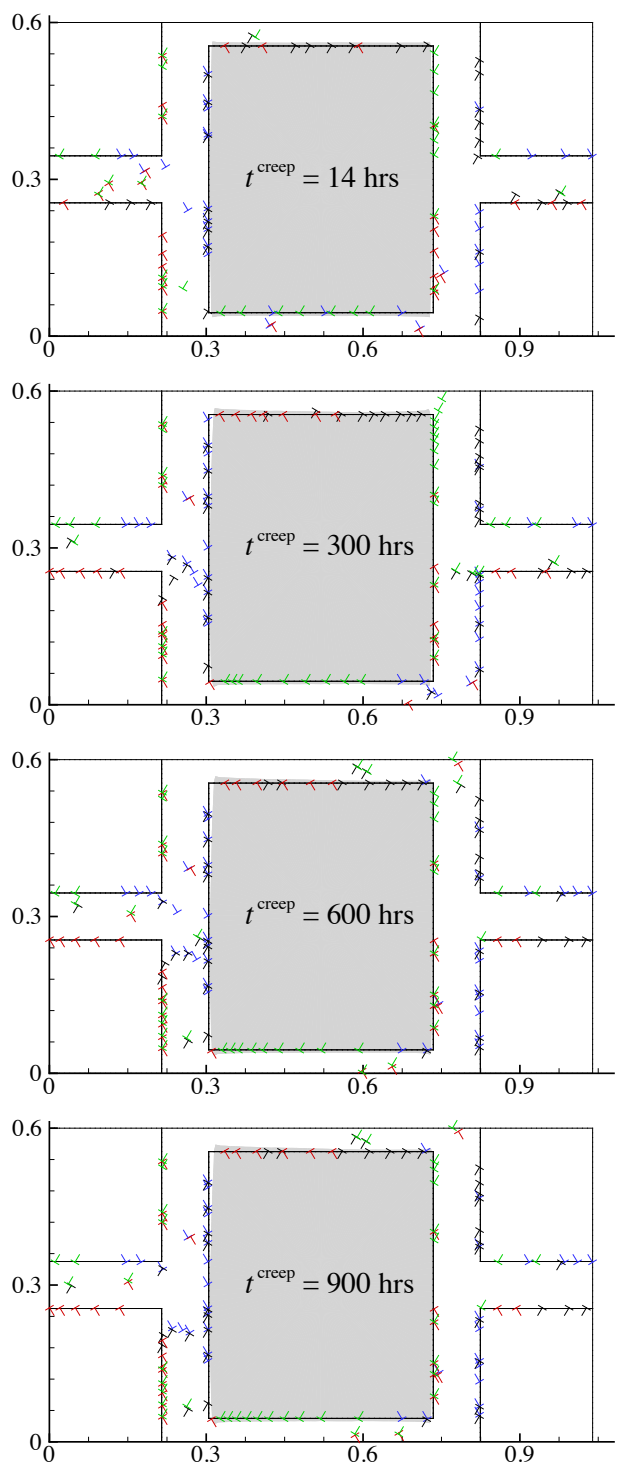

b
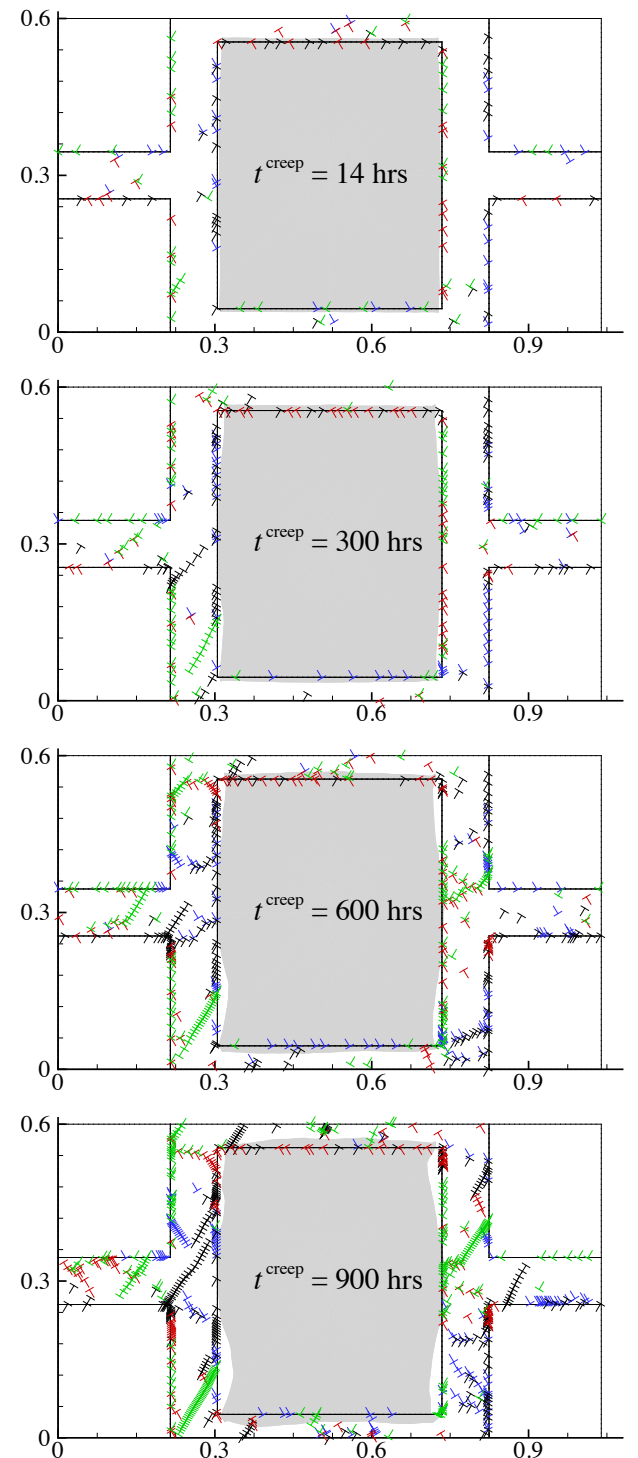

Figure 3: The evolution of dislocation structure in the unit cell of a superalloy with a dislocation source density $\rho_{\text {nuc }}=500 \mu \mathrm{m}^{-2}$ subjected to a normalised applied stress $\bar{\Sigma}=3.8$. The structure is shown at four selected creep times $t^{\text {creep }}$ for the cases with the normalised interfacial diffusion constant (a) $\overline{\mathscr{D}}=0$ and (b) $\overline{\mathscr{D}}=0.1$. The deformation of the central $\gamma^{\prime}$ precipitate is indicated in each case with the displacements magnified by a factor of 6 to aid visualisation and the colours assigned to dislocations are defined in Fig. 1. All dimensions are in $\mu \mathrm{m}$. 

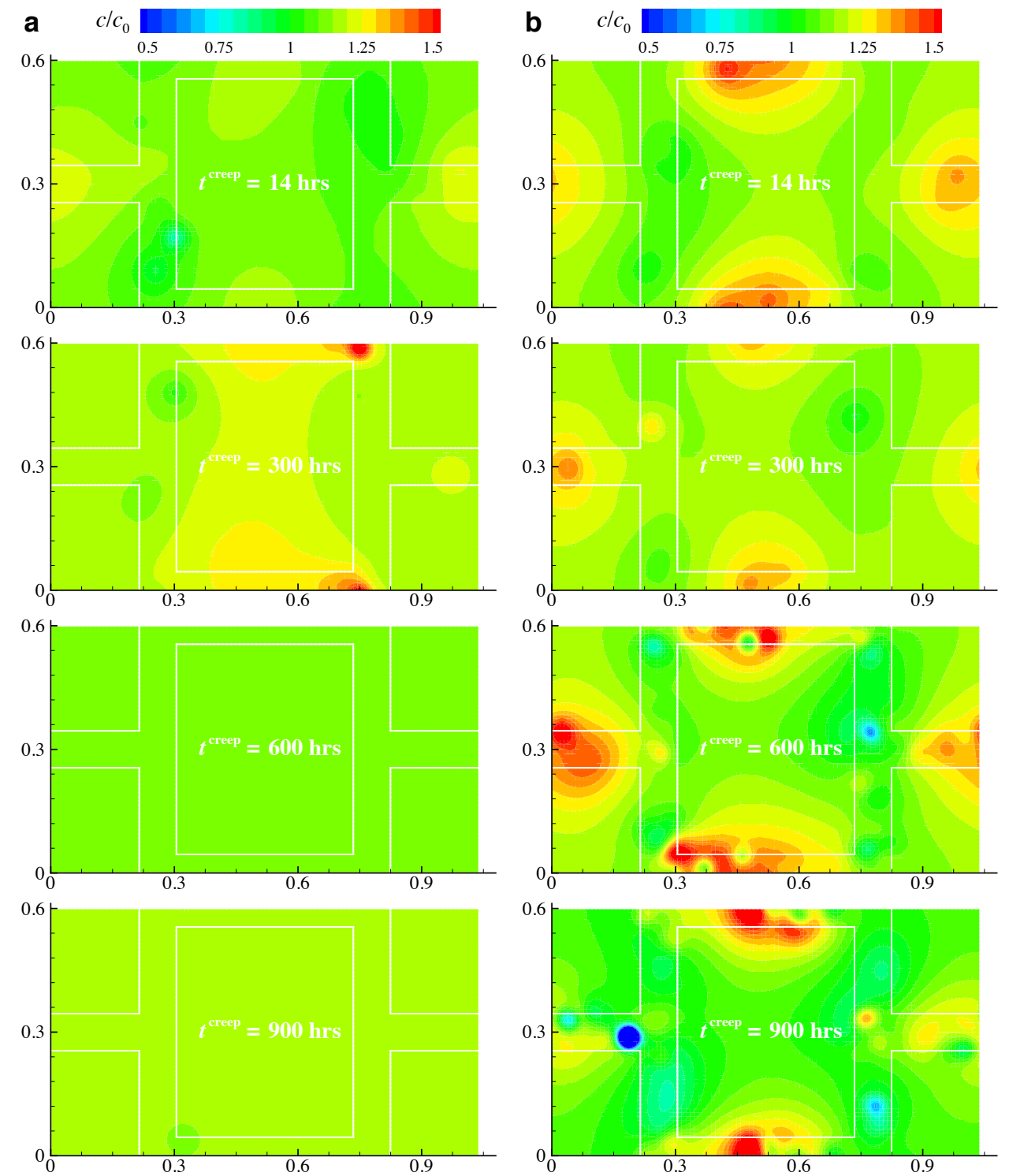

Figure 4: The evolution of the normalised vacancy concentration $c / c_{0}$ in the unit cell of a superalloy with a dislocation source density $\rho_{\text {nuc }}=500 \mu \mathrm{m}^{-2}$ subjected to a normalised applied stress $\bar{\Sigma}=3.8$. The distributions are shown at four selected creep times $t^{\text {creep }}$ for the cases with the normalised interfacial diffusion constant (a) $\overline{\mathscr{D}}=0$ and (b) $\overline{\mathscr{D}}=0.1$. All dimensions are in $\mu \mathrm{m}$. 


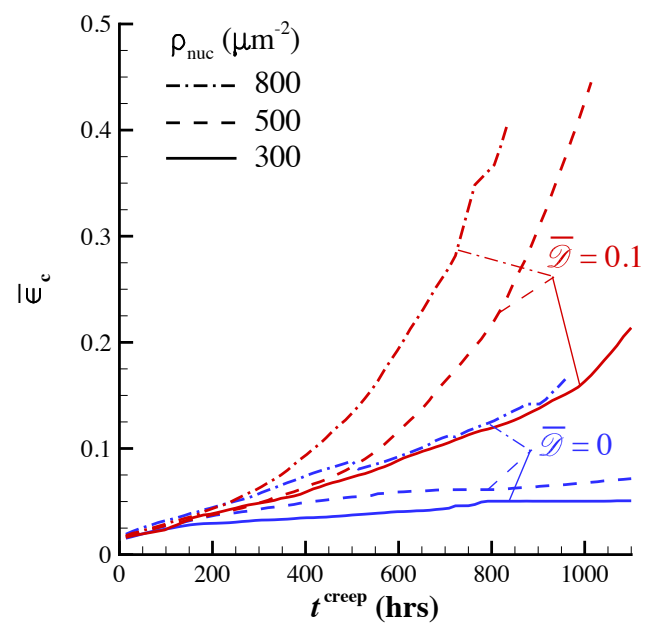

Figure 5: DDP predictions of the variation of the average strain $\bar{\epsilon}_{\mathrm{c}}$ in the $\gamma$ phase due to dislocation climb with the creep time $t^{\text {creep }}$ for the superalloy subjected to a normalised stress $\bar{\Sigma}=3.8$. Results are shown for normalised interfacial diffusion constants $\overline{\mathscr{D}}=0$ and $\overline{\mathscr{D}}=0.1$ and three values of density $\rho_{\text {nuc }}$ of dislocation sources. 

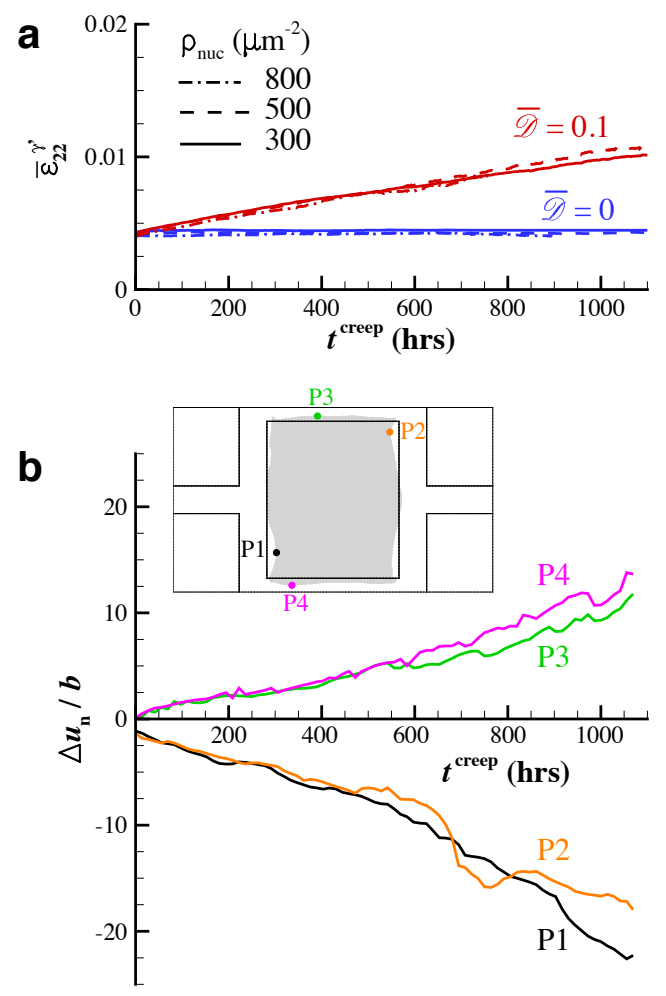

Figure 6: (a) DDP predictions of the temporal variation of the average strain $\bar{\varepsilon}_{22}^{\gamma^{\prime}}$ of the $\gamma^{\prime}$ precipitate with $t^{\text {creep }}$. Results are shown for an applied normalised stress $\bar{\Sigma}=3.8$ for superalloys with normalised interfacial diffusion constants $\overline{\mathscr{D}}=0$ and 0.1 and three values of density $\rho_{\text {nuc }}$ of dislocation sources. (b) The temporal variation of the normalised displacements $\Delta u_{\mathrm{n}} / b$ at four locations (indicated in the inset) on the surface of the central $\gamma^{\prime}$ precipitate of a superalloy with $\rho_{\text {nuc }}=500 \mu \mathrm{m}^{-2}$ and $\overline{\mathscr{D}}=0.1$ subjected to a stress $\bar{\Sigma}=3.8$. 

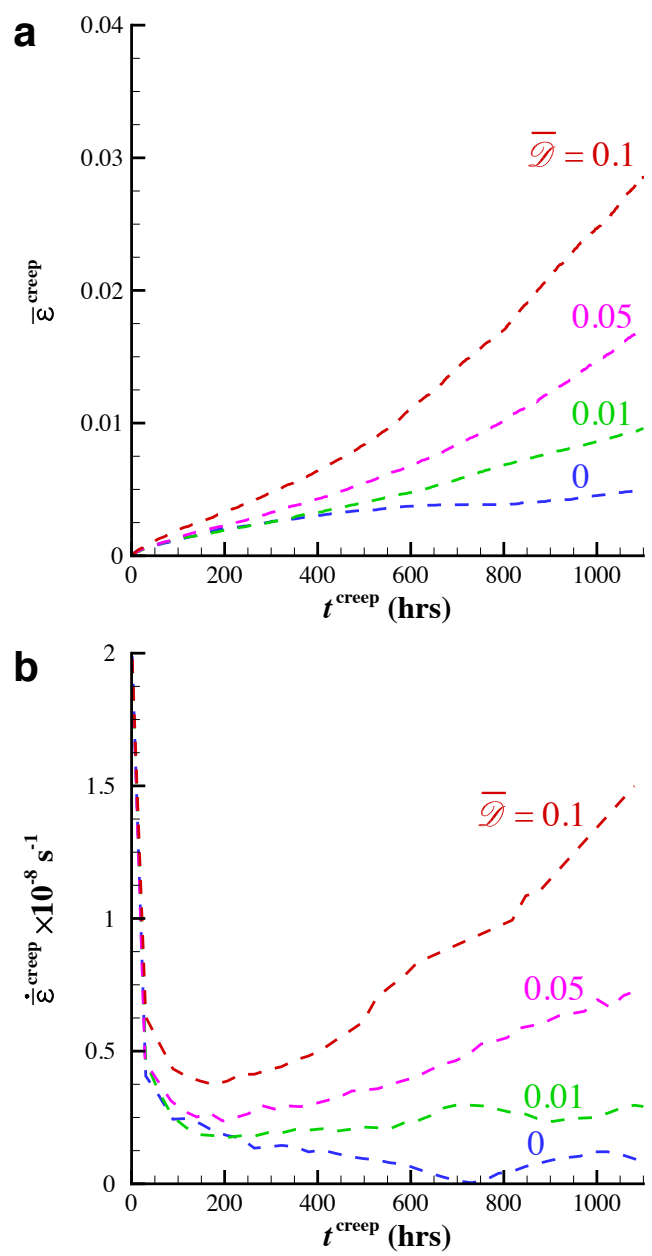

Figure 7: DDP predictions of (a) the creep strain $\bar{\varepsilon}^{\text {creep }}$ versus the creep time $t^{\text {creep }}$ and (b) the corresponding temporal evolution of the creep strain rate $\dot{\bar{\varepsilon}}^{\text {creep }}$ in a superalloy subjected to a normalised tensile stress $\bar{\Sigma}=3.8$ with a density $\rho_{\text {nuc }}=500 \mu \mathrm{m}^{-2}$ of dislocation sources. Results are shown for four selected values of $\overline{\mathscr{D}}$. 

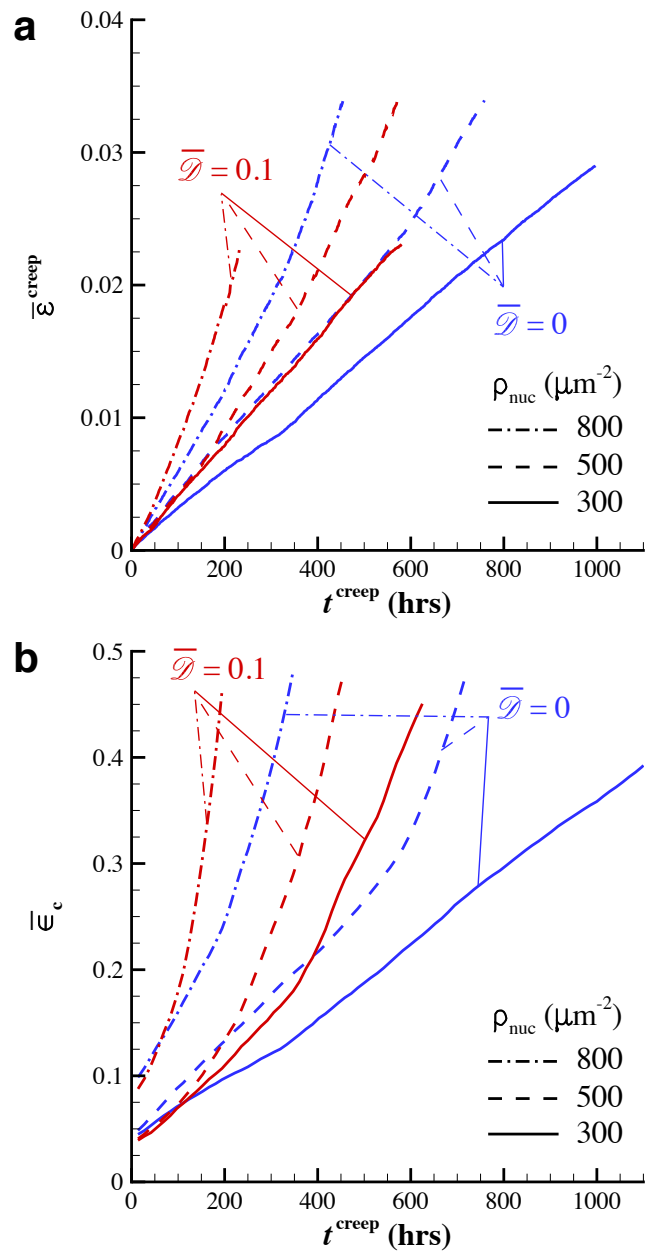

Figure 8: DDP predictions of (a) the creep strain $\bar{\varepsilon}^{\text {creep }}$ versus the creep time $t^{\text {creep }}$ and (b) the corresponding temporal evolution of average strain $\bar{\epsilon}_{\mathrm{c}}$ in the $\gamma$ phase due to dislocation climb. Results are shown for an applied normalised stress $\bar{\Sigma}=6.1$ for superalloys with normalised interfacial diffusion constants $\overline{\mathscr{D}}=0$ and 0.1 and three values of density $\rho_{\text {nuc }}$ of dislocation sources. 

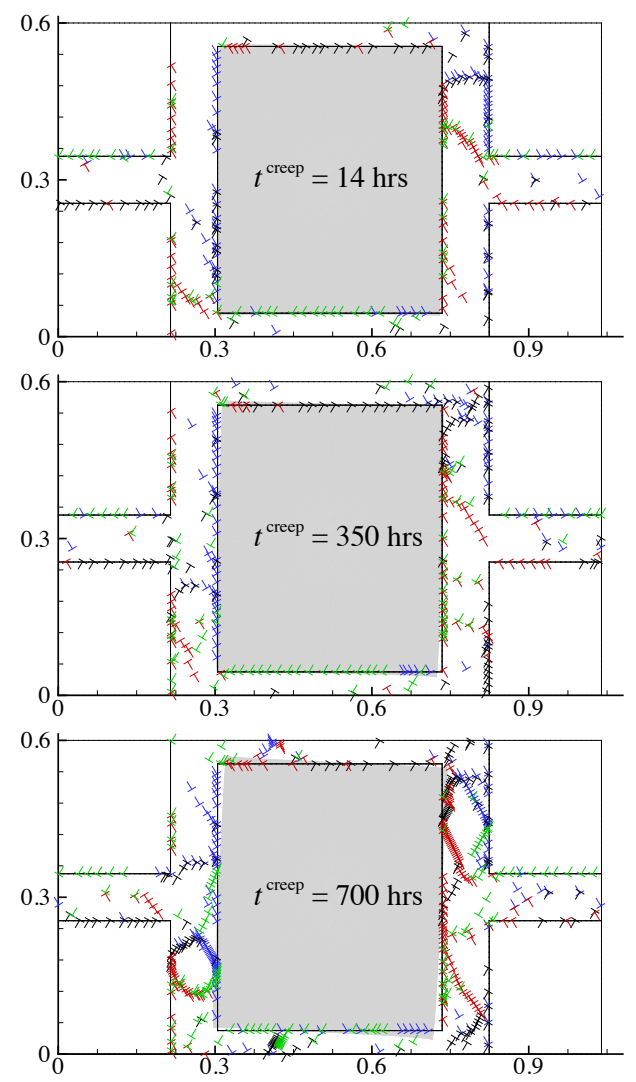

Figure 9: The evolution of dislocation structure in the unit cell of a superalloy with a dislocation source density $\rho_{\text {nuc }}=500 \mu \mathrm{m}^{-2}$ subjected to a normalised applied stress $\bar{\Sigma}=6.1$. The structure is shown at three selected creep times $t^{\text {creep }}$ for the case with no interfacial diffusion $(\overline{\mathscr{D}}=0)$. The deformation of the central $\gamma^{\prime}$ precipitate is indicated in each case with the displacements magnified by a factor of 2 to aid visualisation. The colours assigned to dislocations are defined in Fig. 1. 


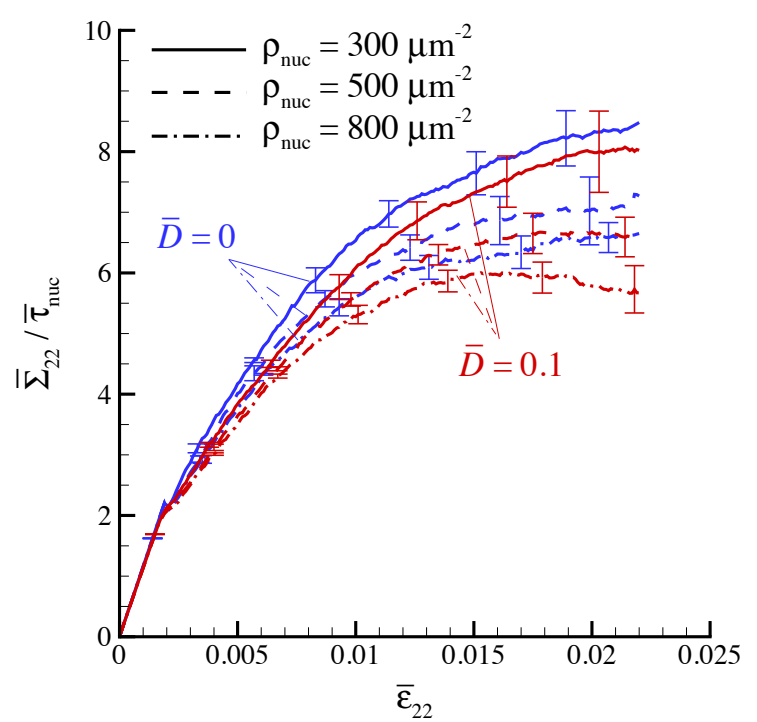

Figure 10: DDP predictions of normalised stress versus strain curves for superalloys subjected to a uniaxial (constant strain rate) loading with normalised interfacial diffusion constants $\overline{\mathscr{D}}=0$ and 0.1 and three values of density $\rho_{\text {nuc }}$ of dislocation sources. 